\title{
Spinor fields classification in arbitrary dimensions and new classes of spinor fields on 7-manifolds
}

\author{
L. Bonora, ${ }^{a}$ K.P.S. de Brito $^{b}$ and Roldão da Rocha ${ }^{a, c}$ \\ ${ }^{a}$ International School for Advanced Studies (SISSA), \\ Via Bonomea 265, 34136 Trieste, Italy \\ ${ }^{b} C C N H$, Universidade Federal do ABC 09210-580, \\ Santo André, SP, Brazil \\ ${ }^{c} C M C C$, Universidade Federal do ABC 09210-580, \\ Santo André, SP, Brazil \\ E-mail: bonora@sissa.it, kelvyn.paterson@ufabc.edu.br, \\ roldao.rocha@ufabc.edu.br
}

ABSTRACT: A classification of spinor fields according to the associated bilinear covariants is constructed in arbitrary dimensions and metric signatures, generalizing Lounesto's 4D spinor field classification. In such a generalized classification a basic role is played by the geometric Fierz identities. In 4D Minkowski spacetime the standard bilinear covariants can be either null or non-null - with the exception of the current density which is invariably different from zero for physical reasons - and sweep all types of spinor fields, including Dirac, Weyl, Majorana and more generally flagpoles, flag-dipoles and dipole spinor fields. To obtain an analogous classification in higher dimensions we use the Fierz identities, which constrain the covariant bilinears in the spinor fields and force some of them to vanish. A generalized graded Fierz aggregate is moreover obtained in such a context simply from the completeness relation. We analyze the particular and important case of Riemannian 7-manifolds, where the Majorana spinor fields turn out to have a quite special place. In particular, at variance with spinor fields in 4D Minkowski spacetime that are classified in six disjoint classes, spinors in Riemannian 7-manifolds are shown to be classified, according to the bilinear covariants: (a) in just one class, in the real case of Majorana spinors; (b) in four classes, in the most general case. Much like new classes of spinor fields in 4D Minkowski spacetime have been evincing new possibilities in physics, we think these new classes of spinor fields in seven dimensions are, in particular, potential candidates for new solutions in the compactification of supergravity on a seven-dimensional manifold and its exotic versions.

KeYwords: Differential and Algebraic Geometry, M-Theory

ARXIV EPRINT: 1411.1590 


\section{Contents}

1 Introduction 1

2 Bilinear covariants in Minkowski space-time $\quad 3$

3 Bilinear forms with spinors in arbitrary dimensions and metric signatures

4 Where are Majorana spinor fields in the generalized spinor field classification? New classes of spinor fields

5 Conclusion

\section{Introduction}

Generalizing Fierz identities in 4D Minkowski spacetime has led to new interesting results on spinor fields, with respect to the textbook ones, and unexpected applications likewise. It is therefore natural to try to do the same in higher dimensions. Fierz identities for formvalued spinor bilinears were considered in arbitrary dimensions and metric signatures [1, 2], using the geometric algebra, being also based in the developments [3, 4]. The most general Fierz identities were further used to construct independent effective four-fermion interactions that contain spin-3/2 chiral fields [5]. Besides, the existence of two natural bilinear forms on the space of spinors were shown in [6] to be related with elements of the exterior algebra. All the Fierz identities can be reduced to a single equation using the extended Cartan map [7]. Reciprocally, spinors are reconstructed from Fierz identities [8] and quantum tomography for Dirac spinors were considered in this context as well [9]. General Fierz identities were further used to find completeness and orthogonality relations [10, 11], where an equivalence between spinor and tensor representations of various quantities have been also constructed. Moreover, Fierz identities are useful to calculate scattering amplitudes [12], being thus employed to calculate electroweak interactions. The inverse problem can be solved likewise by using the Fierz identities [13]. Various Fierz identities are further needed to show the invariance of the $D=11, N=1$ supergravity action under a local supersymmetry transformation, and the supercovariance of the fermion field equation [14]. In supersymmetric gauge theories, supersymmetry constraints imply the existence of certain Fierz identities for real Clifford algebras [15]. These identities hold merely for 2, 4, 8 and 16 supercharges [16]. Fierz identities were also generalized for non-integer dimensions in $[17]$.

The prominent relevance of Fierz identities can be moreover measured by their role on the recent emergence of new kinds of spinor fields. The subject concerning those new 
spinor fields and their applications has been widening, mainly since the middle of the last decade. Fierz identities were used by Lounesto to classify spinor fields in Minkowski spacetime according to the bilinear covariants [18]. Indeed, Lounesto showed that spinor fields can be accommodated in six disjoint classes, that encompass all the spinor fields in Minkowski spacetime. The first three types of spinor fields in such classification are named Dirac spinor fields: this is actually a generalization, as it does not restrict to the standard Dirac spinor field, which is an eigenspinor of the parity operator, providing hence further physical solutions for the Dirac equation. Indeed such three classes of regular spinor fields appear as solutions of the Dirac equation in different contexts. They are characterized by either the scalar or the pseudoscalar (or even both) bilinear covariants being nonzero. The other three classes of (singular) spinors are known as flag-dipole, flagpole and dipole spinor fields, and have both the scalar and pseudoscalar bilinear covariants vanishing. The latter classes contain, besides a rich geometric structure, spinor fields with new dynamics. Flagpole spinor fields have been recently considered in cosmology [19], being explored as candidates for dark matter in various contexts [20-23], wherein Elko and Majorana spinor fields evince prominent roles [24]. Flag-dipole ones are typified for instance by recently found new solutions of the Dirac equation in ESK gravities [25]. Dipole spinor fields include Weyl spinor fields as their most known representative. All the spinor classes have been lately thoroughly characterized [26]. A complete overview of this classification with further applications in field theory and gravitation can be found in [27], being also further explored in the context of black hole thermodynamics [28]. Indeed, black hole tunnelling methods were studied for Elko spinor fields as special type of flagpoles [28], which play an essential role in constructing various theories of gravity naturally arising from supergravity $[29,30]$. Flagpoles spinor fields and Lounesto spinor field classification are moreover discussed in the context of the instanton Hopf fibration [31], and experimental signatures of the type-5 spinors in such a classification are related to the Higgs boson at LHC [32]. An up-to-date overview on a special class of such spinor fields can be found in [33] and references therein.

Fierz identities make it possible to deduce a classification of spinors on the spinor bundle associated to manifolds of arbitrary dimensions, departing from the case proposed by Lounesto, that holds solely for Minkowski spacetime [18]. The aim of the present paper is two-fold: besides generalizing Lounesto's spinor field classification for spacetimes of arbitrary dimension and metric signatures and proposing a graded Fierz aggregate, we focus in particular on the noteworthy case of spinor fields on seven-dimensional manifolds, in particular those on the 7-sphere. This is motivated by their wide physical applications, for instance, in $D=11$ supergravity [34-36]. In fact, spontaneous compactifications of $D=11$ supergravity [34] on Riemannian 7-manifolds are well-known to contain all the degrees of freedom of the massless sector of gauged $N=8$ supergravity theory [36]. Explicit forms of the Killing spinors can be also obtained in the supergravity theory that admits $\mathrm{AdS}_{4} \times S^{7}$ solutions. $S^{7}$ spinors and the Kač-Moody algebra of $S^{7}$ were also considered on the parallelizable 7 -sphere [37, 38].

We use the Fierz identities constraining bilinear covariants constructed by spinor fields in arbitrary dimensions [1], that force some of the respective bilinear covariants to be zero and study the important case of seven dimensions, in particular classifying Majorana spinor 
fields. New kinds of classes of spinor fields are hence obtained, which are hidden if one considers only the real spin bundle on Riemannian 7-manifolds.

This paper is organized as follows: in section II, the bilinear covariants are used to revisit the classification of spinor fields in Minkowski spacetime, according to the Lounesto's classification prescription, and the Fierz aggregate and its related boomerang are defined. In section III, the bilinear covariants associated to spinor fields in arbitrary dimensions and metric signatures are introduced and all the possibilities for them are listed. In section IV, the geometric Fierz identities are employed and from the admissible pairings between spinor fields the number of classes in the spinor field classification are constrained. We study the case of Majorana spinor fields on Riemannian 7-manifolds and define the graded Fierz aggregate as a particular case of the completeness condition. We conclude that Majorana spinors in seven dimensions pertain to solely one class, according the bilinear covariants, as some of these bilinears are identically zero. Spinor fields on these 7-manifolds can be classified in four classes, departing from the classification for the real spin bundle. One of the new classes encompasses the Majorana spinor fields and the others provide new candidates for physical solutions, for instance, in supergravity.

\section{Bilinear covariants in Minkowski space-time}

In order to fix the notation, consider an oriented manifold $(M, g)$, where the metric $g$ has signature $(p, q)$, and its associated tangent [cotangent] bundle $T M\left[T^{*} M\right]$, having sections consisting of $n$-dimensional $(n=p+q)$ real vector spaces. Denoting sections of the exterior bundle by $\sec \bigwedge(T M)$, given a $k$-vector $a \in \sec \bigwedge^{k}(T M)$, the grade involution is defined by $\hat{a}=(-1)^{k} a$ and the reversion by $\tilde{a}=(-1)^{[[k / 2]]} a$, where $[[k]]$ stands for the integral part of $k$. The conjugation is the composition of the two previous morphisms. Moreover, when $g$ is extended from $\sec \bigwedge^{1}(T M)=\sec T^{*} M$ to sec $\bigwedge(T M)$, and by considering $a, b, c \in \sec \bigwedge(V)$, the left [right] contraction can be defined by $g(a\lrcorner b, c)=g(b, \tilde{a} \wedge$ c) $\left[g(a\llcorner b, c)=g(b, a \wedge \tilde{c})]\right.$. The Clifford product between a vector field $v \in \sec \bigwedge^{1}(T M)$ and a multivector $a \in \sec \bigwedge(T M)$ is prescribed by $v \circ a=v \wedge a+v\lrcorner a$. The dual Hodge operator $\star: \sec \bigwedge(T M) \rightarrow \sec \bigwedge(T M)$ is defined by $a \wedge \star b=g(a, b)$. The Grassmann algebra $(\bigwedge(T M), g)$ endowed with the Clifford product is denoted by $C \ell_{p, q}$, the Clifford algebra associated with $\sec \bigwedge^{1}(T M) \simeq \mathbb{R}^{p, q}$.

When the Minkowski spacetime is considered, the set $\left\{e_{\mu}\right\}$ represents sections of the frame bundle $\mathbf{P}_{\mathrm{SO}_{1,3}^{e}}(M)$ and $\left\{\theta^{\mu}\right\}$ is the dual basis $\left\{e_{\mu}\right\}$, namely, $\theta^{\mu}\left(e_{\mu}\right)=\delta_{\nu}^{\mu}$. Classical spinor fields are objects in the carrier space associated to a $\rho=\mathrm{D}^{(1 / 2,0)} \oplus \mathrm{D}^{(0,1 / 2)}$ representation of the Lorentz group, and can be thought as being sections of the vector bundle $\mathbf{P}_{\operatorname{Spin}_{1,3}^{e}}(M) \times_{\rho} \mathbb{C}^{4}$. Moreover, the classical spinor fields carrying either the $\mathrm{D}^{(0,1 / 2)}$ or the $\mathrm{D}^{(1 / 2,0)}$ representation of the Lorentz group are sections of the vector bundle $\mathbf{P}_{\operatorname{Spin}_{1,3}^{e}}(M) \times{ }_{\rho^{\prime}} \mathbb{C}^{2}$, where $\rho^{\prime}$ stands for either the $\mathrm{D}^{(1 / 2,0)}$ or the $\mathrm{D}^{(0,1 / 2)}$ representation of the Lorentz group. Given a spinor field $\psi \in \sec \mathbf{P}_{\operatorname{Spin}_{1,3}^{e}}(M) \times_{\rho} \mathbb{C}^{4}$, the bilinear covariants 
are the following sections of the exterior algebra bundle $\bigwedge(T M)[18,39,40]$ :

$$
\begin{aligned}
\sigma & =\bar{\psi} \psi \\
\mathbf{J} & =J_{\mu} \theta^{\mu}=\bar{\psi} \gamma_{\mu} \psi \theta^{\mu}, \\
\mathbf{S} & =S_{\mu \nu} \theta^{\mu} \wedge \theta^{\nu}=\frac{1}{2} i \bar{\psi} \gamma_{\mu \nu} \psi \theta^{\mu} \wedge \theta^{\nu}, \\
\mathbf{K} & =K_{\mu} \theta^{\mu}=i \bar{\psi} \gamma_{5} \gamma_{\mu} \psi \theta^{\mu}, \\
\omega & =-\bar{\psi} \gamma_{5} \psi,
\end{aligned}
$$

where $\bar{\psi}=\psi^{\dagger} \gamma_{0}, \gamma_{5}:=\gamma_{0} \gamma_{1} \gamma_{2} \gamma_{3}$ and the set $\left\{\mathbf{1}, \gamma_{\mu}, \gamma_{\mu} \gamma_{\nu}, \gamma_{\mu} \gamma_{\nu} \gamma_{\rho}, \gamma_{5}\right\}(\mu<\nu<\rho)$ is a basis for $M(4, \mathbb{C})$ satisfying $\gamma_{\mu} \gamma_{\nu}+\gamma_{\nu} \gamma_{\mu}=2 \eta_{\mu \nu} 1$ and the Clifford product is denoted here by juxtaposition [40].

The space-like 1-form $\mathbf{K}$ designates the spin direction, the 2-form $\mathbf{S}$ denotes the wellknown intrinsic angular momentum, and the time-like 1 -form $\mathbf{J}$ stands for the current of probability. The bilinear covariants satisfy the Fierz identities [18, 39]

$$
-\left(\omega+\sigma \gamma_{5}\right) \mathbf{S}=\mathbf{J} \wedge \mathbf{K}, \quad \mathbf{K}^{2}+\mathbf{J}^{2}=0=\mathbf{J}\left\llcorner\mathbf{K}, \quad \mathbf{J}^{2}=\omega^{2}+\sigma^{2} .\right.
$$

When $\omega=0=\sigma$, a spinor field is said to be singular, and regular otherwise.

Lounesto [18] classified spinor fields into six disjoint classes. In the classes (1), (2), and (3) beneath it is implicit that $\mathbf{J}, \mathbf{K}$ and $\mathbf{S}$ are simultaneously different from zero, and in the classes (4), (5), and (6) just $\mathbf{J} \neq 0$ :
1) $\omega \neq 0, \quad \sigma \neq 0$
4) $\omega=\sigma=0, \quad \mathbf{K} \neq 0, \quad \mathbf{S} \neq 0$
2) $\omega=0, \quad \sigma \neq 0$
5) $\omega=\sigma=0, \quad \mathbf{K}=0, \quad \mathbf{S} \neq 0$
3) $\omega \neq 0, \quad \sigma=0$
6) $\omega=\sigma=0, \quad \mathbf{S}=0, \quad \mathbf{K} \neq 0$

Spinor fields of types-1, -2 , and -3 are called Dirac spinor fields whilst spinor fields of types-4, -5 , and -6 are flag-dipoles, flagpoles and dipole spinor fields, respectively [18]. It is worthwhile to emphasize that the naming "Dirac spinors" in Lounesto's classification is wider than the one adopted in textbooks, where Dirac spinors are eigenstates of the parity operator. The first physical example of flag-dipole spinor fields has been found very recently in [25] as solutions of the Dirac equation in a $f(R)$ background with torsion. Moreover, Majorana and Elko spinor fields reside in the class of spinors of type-5 [24], and Weyl spinor fields are a particular case of a type-6 dipole spinor fields [18], wherein further spinor fields have been scarcely scrutinized. It is also worth to point out that in four and in six dimensions pure spinors coincide with Weyl spinors due to an accident in these dimensions $[18,31]$, while there are quadratic constraints that pure spinors obey in higher dimensions [41]. In particular, the constraints in ten dimensions play an important role in Berkovits' approach to superstrings [42, 43].

A multivector field [18]

$$
Z=\omega \gamma_{5}+i \mathbf{K} \gamma_{5}+i \mathbf{S}+\mathbf{J}+\sigma
$$


is called a Fierz aggregate when $\omega, \mathbf{S}, \mathbf{K}, \mathbf{J}, \sigma$ fulfil the Fierz identities (2.2). Additionally, if $\gamma^{0} Z \gamma^{0}=Z^{\dagger}$, the Fierz aggregate is named a boomerang [18]. When singular spinor fields are scrutinized, the Fierz identities are replaced by the most assorted expressions [39]:

$$
\begin{aligned}
Z \gamma_{\mu} Z & =4 J_{\mu} Z, & Z^{2} & =4 \sigma Z, \\
-Z \gamma_{5} Z & =4 \omega Z, & i Z \gamma_{5} \gamma_{\mu} Z & =4 K_{\mu} Z .
\end{aligned}
$$

\section{Bilinear forms with spinors in arbitrary dimensions and metric signa- tures}

Going to arbitrary dimensions, one starts from the spin bundle $S$ associated to a manifold $(M, g)$. A crucial role is played by the Kähler-Atiyah bundle $(\sec \wedge(T M), \circ)$, where the Clifford product shall be denoted by $\circ$. The spin bundle $S$ is defined upon the even Kähler-Atiyah bundle $(\bigwedge(T M), \circ)$ and has module structure specified by a morphism $\gamma$ : $(\bigwedge(T M), \circ) \rightarrow(\operatorname{End}(S)$, o $)$ (for more details, see, e. g., [1, 2, 40, 44-46]). In addition, a direct sum decomposition $S=S_{0} \oplus S_{1}$ is provided by an idempotent endomorphism R $\in \Gamma(\operatorname{End}(S))($ here $\Gamma(\operatorname{End}(S))$ denotes the space of smooth sections of $\operatorname{End}(S))$ - which for some dimensions and signatures is usually identified with the volume element $\gamma^{n+1}[1]$. The sub-bundles $S_{0}$ and $S_{1}$ are determined by the eigenvalues \pm 1 of $\mathrm{R}$, and the above direct sum decomposition is said to be non-trivial if both $S_{0}$ and $S_{1}$ are different from zero. This is equivalent to saying that the Clifford algebras $\mathcal{C} \ell_{p, q}$ constructed on the cotangent bundle on each point of $M$ are universal [47]. The restriction $\dot{\gamma}: \sec \bigwedge(T M) \rightarrow \operatorname{End}(S)$ is named spin endomorphism if it commutes with $\gamma(\xi)$, for all $\xi \in \sec \bigwedge(T M)$ [1].

Spin projectors are defined by $\Pi_{ \pm}=\frac{1}{2}(I \pm \mathrm{R})$, where $I$ denotes the identity operator on $S$, providing the direct sum $S=S^{+} \oplus S^{-}$, where $S^{ \pm}=\Pi_{ \pm}(S)$. The sections of $S^{ \pm}$are called [symplectic] Majorana-Weyl spinors when $p-q \equiv 0 \bmod 8[p-q \equiv 4 \bmod 8]$, while the sections of $S^{+}$are known as [symplectic] Majorana spinors when $p-q \equiv 7 \bmod 8$ $[p-q \equiv 6 \bmod 8]$. Classical spinors $S_{p, q}$ of the Clifford bundle of $M$ are well-known to be elements of the irreducible representation space of the component of the group $\operatorname{Spin}(p, q)$ connected to the identity, and their classification can be summarized in table 1.

The ring of quaternions is denoted by $\mathbb{H}$. For the complex case the classification is well-known to be simpler, being evinced by table 2 .

Now consider the complex structure $J \in \Gamma(\operatorname{End}(S))$, which in particular is given by $J= \pm \gamma^{n+1}= \pm \gamma^{1} \mathbf{o} \cdots \mathbf{o} \gamma^{n}$ when $p-q \equiv 3,7 \bmod 8$ [1], and an endomorphism $D$ on the spin bundle that satisfies for all $\xi \in \sec \bigwedge(T M)$ the following expressions $[1,48]$

$$
D \circ D=(-1)^{\frac{1+p-q}{4}} I, \quad D \circ \gamma(\xi)=\gamma(\hat{\xi}) \circ D .
$$

Such expressions are taken into account hereupon, being essential to define both the classification of spinor fields and the graded Fierz aggregate as well.

Starting from an orthonormal local coframe $\left\{e^{a}\right\}_{a=1}^{n} \subset \mathbf{P}_{\mathrm{SO}_{p, q}^{e}}(M)$, recall from [49] that a non-degenerate bilinear pairing $B$ on the spin bundle $S$ is named admissible if the following requirements hold: a) $B$ is either symmetric or skew-symmetric; b) if $p-$ $q \equiv 0,4,6,7 \bmod 8$, then $S^{+}$and $S^{-}$are either isotropic or orthogonal with respect to 


\begin{tabular}{|c|c|c|c|c|}
\hline $\begin{array}{c}p-q \\
\bmod 8\end{array}$ & 0 & 1 & 2 & 3 \\
\hline$S_{p, q}$ & $\begin{array}{c}\oplus \\
\mathbb{R}^{2^{[(n-1) / 2]}} \\
\mathbb{R}^{[(n-1) / 2]}\end{array}$ & $\mathbb{R}^{\left[^{[(n-1) / 2]}\right.}$ & $\mathbb{C}^{2^{[(n-1) / 2]}}$ & $\mathbb{H}^{2^{[(n-1) / 2]-1}}$ \\
\hline $\begin{array}{c}p-q \\
\bmod 8\end{array}$ & 4 & 5 & 6 & 7 \\
\hline$S_{p, q}$ & $\begin{array}{c}\oplus \\
\mathbb{H}^{[(n-1) / 2]-1}\end{array}$ & $\mathbb{H}^{2^{[(n-1) / 2]-1}}$ & $\mathbb{C}^{2^{[(n-1) / 2]}}$ & $\mathbb{R}^{2^{[(n-1) / 2]}}$ \\
\hline
\end{tabular}

Table 1. Classical Spinors Classification table - Real case $(p+q=n)$.

\begin{tabular}{|c|c|}
\hline$n=2 k$ & $\mathbb{C}^{2^{k-1}} \oplus \mathbb{C}^{2^{k-1}}$ \\
\hline$n=2 k+1$ & $\mathbb{C}^{2^{k}}$ \\
\hline
\end{tabular}

Table 2. Classical Spinors Classification table - Complex case.

$B$ [1]; c) for any $\xi \in \sec \bigwedge(T M)$ one has the transpose relation $\gamma(\xi)^{\top}=\gamma(\xi)$ if and only if $B\left(\gamma(\xi) \psi, \psi^{\prime}\right)=B\left(\psi, \gamma(\xi) \psi^{\prime}\right)$, where $\xi$ stands for either the usual reversion $\tilde{\xi}$, if $B$ is symmetric, or the Clifford conjugation $\tilde{\bar{\xi}}$ otherwise.

A more general pairing can be taken into account, by complexifying its restriction to the real bundle $S^{+}$. In fact, by adopting hereon the notation $\psi, \psi^{\prime} \in \Gamma(S)$, where $\Gamma(S)$ denotes the space of smooth sections of the spin bundle $S$, the bilinear pairing $\beta_{0}$ on $S$ is obtained [1]

$$
\begin{aligned}
\beta_{0}\left(\psi, \psi^{\prime}\right)=B\left({ }^{(\mathrm{Re})} \psi,{ }^{(\mathrm{Re})} \psi^{\prime}\right)-B\left({ }^{(\mathrm{Im})} \psi\right. & \left.,{ }^{(\mathrm{Im})} \psi^{\prime}\right) \\
+ & i\left[B\left({ }^{(\mathrm{Re})} \psi,{ }^{(\mathrm{Im})} \psi^{\prime}\right)+B\left({ }^{(\mathrm{Im})} \psi,{ }^{(\mathrm{Re})} \psi^{\prime}\right)\right],
\end{aligned}
$$

where ${ }^{(\operatorname{Re})} \psi=\frac{1}{2}(\psi+D(\psi))$ and ${ }^{(\operatorname{Im})} \psi=\frac{1}{2}(\psi-D(\psi))$ are the real and imaginary parts of $\psi$, respectively [1].

For an ordered set of indexes $\left(\alpha_{1}, \ldots, \alpha_{k}\right), 1 \leq \alpha_{k} \leq n=p+q$, the notation $e^{\alpha_{1} \ldots \alpha_{k}}=$ $e^{\alpha_{1}} \wedge \cdots \wedge e^{\alpha_{k}}$ and $\gamma^{\alpha_{1} \ldots \alpha_{k}}=\gamma^{\alpha_{1}} \mathbf{o} \cdots \mathbf{o} \gamma^{\alpha_{k}}$ shall be adopted hereupon, with analogous expressions for their respective contravariant counterparts. The product "o" may be also denoted by juxtaposition, being used explicitly solely when we want to emphasize the underlying structure.

In the previous section the usual spinor conjugate $\bar{\psi}=\psi^{\dagger} \gamma^{0}$ represents the spinor dual to $\psi$. In Minkowski spacetime it has this form in order to produce a Lorentz invariant quantity. In arbitrary dimensions it can have a much more general form, which plays a prominent role in the framework of bilinear forms. In fact, a spin-invariant product can always be written as [47]

$$
\beta\left(\psi, \psi^{\prime}\right)=a^{-1} \tilde{\psi} \psi^{\prime}=\psi^{\dagger} a^{-1} \psi^{\prime}
$$


where $\psi, \psi^{\prime}$ are spinor fields and $a \in \Gamma(\operatorname{End}(S))$. Since $\tilde{a}=a$ and $\stackrel{\circ}{b}=a b^{\dagger} a^{-1}$, where ${ }^{\circ}$ indicates an arbitrary adjoint involution, then $a^{\dagger}=a[47]$. In this way, the spinor conjugation can be written more generally as $\bar{\psi}=a^{-1} \tilde{\psi}=\psi^{\dagger} a^{-1}$, and we can use this definition to write the most general bilinear on $S$ :

$$
\beta_{k}\left(\psi, \psi^{\prime}\right)=B\left(\psi, \gamma_{\alpha_{1} \ldots \alpha_{k}} \psi^{\prime}\right)=\bar{\psi} \gamma_{\alpha_{1} \ldots \alpha_{k}} \psi^{\prime}
$$

On the spin bundle $S$, bilinear covariants are thus more generally defined as follows, given $\psi \in S$ :

$$
\begin{aligned}
\Omega_{1} & =\bar{\psi} \psi \\
J_{\alpha_{1}} & =\bar{\psi} \gamma_{\alpha_{1}} \psi \\
S_{\alpha_{1} \alpha_{2}} & =\bar{\psi} \gamma_{\alpha_{1} \alpha_{2}} \psi \\
& \vdots \\
S_{\alpha_{1} \ldots \alpha_{d+1}} & =\bar{\psi} \gamma_{\alpha_{1} \ldots \alpha_{d+1}} \psi, \quad d<k-1 \\
& \vdots \\
S_{\alpha_{1} \ldots \alpha_{k}} & =\bar{\psi} \gamma_{\alpha_{1} \ldots \alpha_{k}} \psi \\
K_{\alpha_{1} \ldots \alpha_{l-1}} & =\bar{\psi} \gamma_{\alpha_{1} \ldots \alpha_{l-1}} \gamma_{n+1} \psi \\
& \vdots \\
K_{\alpha_{1} \ldots \alpha_{m}} & =\bar{\psi} \gamma_{\alpha_{1} \ldots \alpha_{m}} \gamma_{n+1} \psi, \quad m<l-1 \\
& \vdots \\
K_{\alpha_{1}} & =\bar{\psi} \gamma_{\alpha_{1}} \gamma_{n+1} \psi \\
\Omega_{2} & =\bar{\psi} \gamma_{n+1} \psi .
\end{aligned}
$$

These bilinear covariants are used to defined the corresponding Fierz aggregate

$$
\begin{aligned}
\mathrm{Z}=\Omega_{1}+ & J_{\mu} \gamma^{\mu}+S_{\mu_{1} \mu_{2}} \gamma^{\mu_{1} \mu_{2}}+\cdots+S_{\mu_{1} \ldots \mu_{p}} \gamma^{\mu_{1} \ldots \mu_{p}} \\
& +K_{\alpha_{1} \ldots \alpha_{q-1}} \gamma^{\alpha_{1} \ldots \alpha_{q-1}} \gamma_{n+1}+\cdots+K_{\mu} \gamma^{\mu} \gamma_{n+1}+\Omega_{2} \gamma_{n+1}
\end{aligned}
$$

that reduces to the standard Fierz aggregate (2.3) when $n=4$. Similarly to the case on Minkowski spacetime, when $\gamma^{0} \mathrm{Z} \gamma^{0}=\mathrm{Z}^{\dagger}$ the above generalized Fierz aggregate will be called a generalized boomerang.

In the next section we shall see that a graded Fierz aggregate can be evinced exclusively from the completeness relation. In addition, most of such bilinear covariants that define it will be shown to be null, as a consequence of constraints imposed by the geometric Fierz identities [1]. It forces the number of spinor field classes to be reduced, being obstructed by the geometric Fierz identities. We will see in the next section that the $4 \mathrm{D}$ Fierz identities for regular spinor fields - given by (2.2) — and for any kind of spinor fields - provided by $(2.4)$ - can be generalized to the geometric Fierz identities in arbitrary dimensions [1], and new classes of Fermi fields on 7-manifolds can be evinced. 


\section{Where are Majorana spinor fields in the generalized spinor field clas- sification? New classes of spinor fields}

When $p-q \equiv 7 \bmod 8$, the endomorphism $D$ is a real structure that defines the complex conjugate via $D(\psi)={ }^{(\operatorname{Im})} \psi$, and can be identified to the spin endomorphism R discussed in section 3 [1]. The projectors $P_{ \pm}=\frac{1}{2}(I \pm D)$ are hence responsible to split a spinor field in real and imaginary components given by respectively by $P_{ \pm}(\psi)=\psi^{ \pm}$. The real vector bundles $S^{ \pm} \equiv P_{ \pm}(S)$ are thus used to identify the spin bundle $S=S^{+} \oplus S^{-}$to the complexification of the real bundle $S^{+}$of Majorana spinor fields [1]. In particular when $n=7$, other useful pairings can be defined from a basic admissible pairing, denoted hereon for the sake of simplicity by $B$, as [1]

$$
B^{\prime}:=B \mathbf{o}(I \otimes J), \quad B^{\prime \prime}:=-B \mathbf{o}[I \otimes(J \mathbf{o} D)], \quad B^{\prime \prime \prime}:=B \mathbf{o}(I \otimes D) .
$$

We can use some of them to define the bilinears (3.2) and (3.3). However, might the higher dimensional analogues of Lounesto's classes of spinor fields provide indeed a different spinor fields classification? We shall answer in an affirmative way this question and discuss later such new possibilities, showing that at least two choices in (4.1) are equivalent under Hodge duality.

When one chooses $\psi$ to be a Majorana spinor, the non null bilinear pairings can be reduced through the fact that $B\left(\psi, \gamma^{\alpha_{1} \ldots \alpha_{k}} \psi\right)=0$ except if $k$ is even [1]. Given any admissible bilinear pairing $B$ on $S$, the endomorphisms $A_{\psi \mid \psi^{\prime}}$ of the spin bundle $S$ have been defined in [1]:

$$
A_{\psi_{1} \mid \psi_{2}}(\psi):=B\left(\psi, \psi_{2}\right) \psi_{1}, \quad \text { for all } \quad \psi, \psi_{1}, \psi_{2} \in \Gamma(S),
$$

and play an important role to determine the geometric Fierz identities, encrypted in the expressions

$$
A_{\psi_{1} \mid \psi_{2}} \text { o } A_{\psi_{3} \mid \psi_{4}}=B\left(\psi_{3}, \psi_{2}\right) A_{\psi_{1} \mid \psi_{4}},
$$

as shall be briefly reviewed in the sequel [1].

Consider now the completeness relation

$$
A_{\psi \mid \psi^{\prime}}=\frac{\ell}{2^{n}} \sum_{k} \frac{1}{k !}(-1)^{k} B\left(\psi, \gamma_{\alpha_{1} \ldots \alpha_{k}} \psi^{\prime}\right) e^{\alpha_{1} \ldots \alpha_{k}},
$$

where either $\ell=2^{\llbracket \frac{n}{2} \rrbracket}$, if $p-q=0,1,2$, or $\ell=2^{\llbracket \frac{n}{2} \pi+1}$ otherwise, where $\llbracket \frac{n}{2} \rrbracket \equiv \frac{n(n-1)}{2}$ mod 2. This expression mimics the Fierz aggregate (2.3). Moreover, every element in the space $\Gamma(\operatorname{End}(S))$, in particular the $A_{\psi \mid \psi^{\prime}}$, can be split uniquely as $A_{\psi \mid \psi^{\prime}}=D \mathbf{o} A_{\psi \mid \psi^{\prime}}^{1}+$ $A_{\psi \mid \psi^{\prime}}^{0}[1]$, where

$$
\begin{aligned}
& A_{\psi \mid \psi^{\prime}}^{0}=\frac{\ell}{2^{n}} \sum_{k} \frac{(-1)^{k}}{k !} B\left(\psi, \gamma_{\alpha_{1} \ldots \alpha_{k}} \psi^{\prime}\right) e^{\alpha_{1} \ldots \alpha_{k}} \\
& A_{\psi \mid \psi^{\prime}}^{1}=\frac{\ell}{2^{n}} \sum_{k} \frac{1}{k !}(-1)^{\left(k+\frac{1+p-q}{4}\right)} B\left(\psi, D \mathbf{o} \gamma_{\alpha_{1} \ldots \alpha_{k}} \psi^{\prime}\right) e^{\alpha_{1} \ldots \alpha_{k}} .
\end{aligned}
$$


The Fierz identities (2.2) — for regular spinor fields in Minkowski spacetime - or more generally (2.4) - for any kind of spinor fields in Minkowski spacetime - can be generalized for arbitrary dimensions, being hence provided by [1]:

$$
\begin{array}{r}
\widehat{A_{\psi_{1} \mid \psi_{2}}^{0}} \circ A_{\psi_{3} \mid \psi_{4}}^{1}+A_{\psi_{1} \mid \psi_{2}}^{1} \circ A_{\psi_{3} \mid \psi_{4}}^{0}=B\left(\psi_{3}, \psi_{2}\right) A_{\psi_{1} \mid \psi_{4}}^{1}, \\
A_{\psi_{1} \mid \psi_{2}}^{0} \circ A_{\psi_{3} \mid \psi_{4}}^{0}+(-1)^{\frac{1+p-q}{4}} \widehat{A_{\psi_{1} \mid \psi_{2}}^{1} \circ A_{\psi_{3} \mid \psi_{4}}^{1}}=B\left(\psi_{3}, \psi_{2}\right) A_{\psi_{1} \mid \psi_{4}}^{0} .
\end{array}
$$

The notation for both elements in $\sec \bigwedge^{k}(T M)$

$$
\begin{aligned}
& A_{\psi \mid \psi^{\prime}}^{0, k}=\frac{1}{k !}(-1)^{k} B\left(\psi, \gamma_{\alpha_{1} \ldots \alpha_{k}} \psi^{\prime}\right) e^{\alpha_{1} \ldots \alpha_{k}} \\
& A_{\psi \mid \psi^{\prime}}^{1, k}=\frac{1}{k !}(-1)^{\frac{1+p-q}{4}+k} B\left(\psi, D \mathbf{o} \gamma_{\alpha_{1} \ldots \alpha_{k}} \psi^{\prime}\right) e^{\alpha_{1} \ldots \alpha_{k}},
\end{aligned}
$$

will be employed accordingly, in order to make it possible to write $A_{\psi \mid \psi^{\prime}}^{\lambda}=\frac{\ell}{2^{n}} \sum_{k} A_{\psi \mid \psi^{\prime}}^{\lambda, k}$, for $\lambda=0,1$.

As we are interested in determining the nature of Majorana spinor fields according to bilinear covariants in 7-manifolds, we focus in the particular, however important case [37] of $n=p+q=7+0$. The case of a Majorana spinor on a Riemannian 7-manifold arises, for example, in the of $N=1$ compactifications of $M$-theory on 7-manifolds [50-52], permitting a geometric characterization by means of the reduction of the structure group of $\sec \bigwedge^{1}(T M)$ from the orthogonal group $\mathrm{O}(7)$ to the exceptional one $\mathrm{G}_{2}$ [1].

Moreover, the expression

$$
\varphi_{k}:=\left|A_{\psi \mid \psi}^{0, k}\right|=\frac{1}{k !} B\left(\psi, \gamma_{\alpha_{1} \ldots \alpha_{k}} \psi\right) e^{\alpha_{1} \ldots \alpha_{k}}
$$

equals zero except if $k$ is even. Together with the symmetry of $B$, this shows that the element $A^{0, k} \equiv A_{\psi \mid \psi}^{0, k}$ vanishes except if $k=0,3,4,7$. Combining this with (4.7), it implies that, for $\psi$ a Majorana spinor, the forms $\varphi_{k}$ equal zero except when $k=0$ or $k=4$. By regarding $\psi$ normalized such that $B(\psi, \psi)=1$, it follows that $A^{0,0}=1$ and the following bilinear can be defined [1]:

$$
\varphi_{4}=\frac{1}{4 !} B\left(\psi, \gamma_{\alpha_{1} \alpha_{2} \alpha_{3} \alpha_{4}} \psi\right) e^{\alpha_{1} \alpha_{2} \alpha_{3} \alpha_{4}}
$$

which are the components of the first generator $A^{0}=\frac{1}{16}\left(1+\varphi_{4}\right)$ of the so called Fierz algebra represented in (4.4a), (4.4b) [1], where $A^{\lambda} \equiv A_{\psi \mid \psi}^{\lambda}$. Moreover, since $A^{1}=A^{0}$, then the Fierz identities (4.5a), (4.5b) are concomitantly equivalent and imply that $\left(\varphi_{4}+1\right) \circ\left(\varphi_{4}+1\right)=$ $8\left(\varphi_{4}+1\right)[1]$.

Now, recall that the product $\Delta_{k}: \sec \bigwedge(T M) \times \sec \bigwedge(T M) \rightarrow \sec \bigwedge(T M)$ is defined iteratively by

$$
\left.\left.\chi \Delta_{k+1} \vartheta=\frac{1}{k+1} g^{a b}\left(e_{a}\right\lrcorner \chi\right) \Delta_{k}\left(e_{b}\right\lrcorner \vartheta\right), \quad \chi, \vartheta \in \sec \bigwedge(T M),
$$

where $g^{a b}$ denotes the metric tensor coefficients. By fixing $\Delta_{0}=\wedge$ as being the exterior product, the next terms are for instance provided by

$$
\begin{aligned}
& \left.\left.\chi \Delta_{1} \vartheta=g^{a b}\left(e_{a}\right\lrcorner \chi\right) \wedge\left(e_{b}\right\lrcorner \vartheta\right), \\
& \left.\left.\left.\left.\chi \Delta_{2} \vartheta=\frac{1}{2} g^{a b} g^{c d}\left[e_{a}\right\lrcorner\left(e_{c}\right\lrcorner \chi\right)\right] \wedge\left[e_{b}\right\lrcorner\left(e_{d}\right\lrcorner \vartheta\right)\right] .
\end{aligned}
$$


When the Clifford product is written as $\varphi_{4} \circ \varphi_{4}=\left\|\varphi_{4}\right\|^{2}-\varphi_{4} \Delta_{2} \varphi_{4}$, the Fierz identities correspond to the following conditions [1]:

$$
\varphi_{4} \Delta_{2} \varphi_{4}=-6 \varphi_{4}, \quad\left\|\varphi_{4}\right\|^{2}=7 .
$$

They are the root to establish the spinor fields classification according to the bilinear covariants.

In the case here to be analyzed $n=p+q=7$, we already know that $\varphi_{k}=0$ except for the values $k \in\{0,3,4,7\}$. In addition, due to the restriction

$$
B\left(\psi, J \mathbf{o} \gamma_{\alpha_{1} \ldots \alpha_{k}} \psi\right)=-B\left(\psi, J \mathbf{o} D \mathbf{o} \gamma_{\alpha_{1} \ldots \alpha_{k}} \psi\right)=0 \text { except it } k=2 j+1, k \in \mathbb{Z},
$$

we obtain that unless $k=3$ or $k=7$ the form $B^{\prime}\left(\psi, \gamma_{\alpha_{1} \ldots \alpha_{k}} \psi\right)$ vanishes. Hence, alternatively we could have chosen any other of the bilinear pairings in (4.1) in order to define the bilinear covariants. For instance, choosing $B^{\prime}$ yields the following definition:

$$
\check{\varphi}_{k}=\frac{1}{k !} B\left(\psi, J \mathbf{o} \gamma_{\alpha_{1} \ldots \alpha_{k}} \psi\right) e^{\alpha_{1} \ldots \alpha_{k}} \in \sec \bigwedge^{k}(T M) .
$$

However, the Hodge duality $\star \xi=\tilde{\xi} J$, where $J=\gamma^{n+1}$, for our case $n=7$ implies that the alternative homogeneous forms

$$
\begin{aligned}
& \check{\varphi}_{3}=\frac{1}{3 !} B\left(\psi, J \mathbf{o} \gamma_{\alpha_{1} \alpha_{2} \alpha_{3}} \psi\right) e^{\alpha_{1} \alpha_{2} \alpha_{3}} \\
& \check{\varphi}_{7}=\frac{1}{7 !} B\left(\psi, J \mathbf{o} \gamma_{\alpha_{1} \ldots \alpha_{7}} \psi\right) e^{\alpha_{1} \ldots \alpha_{7}}
\end{aligned}
$$

do not vanish likewise. A similar reasoning [1] implies that the other bilinear pairings in (4.1) contain no information besides the ones provided by (4.7). The Fierz identities take now the form

$$
\check{\varphi}_{3} \Delta_{i} \varphi_{4}=0 \quad(i=1,3), \quad \check{\varphi}_{3} \wedge \varphi_{4}=\left\|\check{\varphi}_{3}\right\|^{2} \gamma^{8} .
$$

Since $\check{\varphi}_{3}=\star \varphi_{4}$ implies that $\left\|\check{\varphi}_{3}\right\|=\left\|\varphi_{4}\right\|$, eq. (4.10) asserts that the 3 -form $\check{\varphi}_{3}$ is non null likewise, and a similar reasoning implies that also $\check{\varphi}_{7} \neq 0$, as $\star 1=\check{\varphi}_{7}$.

Hence, only the bilinears

$$
\begin{aligned}
\varphi_{0} & =B(\psi, \psi) \\
\varphi_{4} & =\frac{1}{4 !} B\left(\psi, \gamma_{\alpha_{1} \alpha_{2} \alpha_{3} \alpha_{4}} \psi\right) e^{\alpha_{1} \alpha_{2} \alpha_{3} \alpha_{4}}
\end{aligned}
$$

are non null. Eq. (4.16) is the higher dimensional analogue of its Minkowski spacetime version provided by eq. (2.1a). Thus the Fierz identities (4.10) imply, in particular, that $\varphi_{4} \neq 0$ and only one type of Majorana spinor results according to a generalized spinor field classification:

$$
\varphi_{0} \neq 0 \in \sec \bigwedge^{0}(T M), \quad \varphi_{4} \neq 0 \in \sec \bigwedge^{4}(T M)
$$


In fact, such class of Majorana spinor fields according to the bilinears in the Clifford bundle $\mathcal{C} \ell_{7,0}$ is provided by:

$$
\varphi_{0} \neq 0, \quad \varphi_{1}=0, \quad \varphi_{2}=0, \quad \varphi_{3}=0, \quad \varphi_{4} \neq 0, \quad \varphi_{5}=0, \quad \varphi_{6}=0, \quad \varphi_{7}=0,
$$

or equivalently, if eq. (4.12) is taken into account,

$$
\check{\varphi}_{7} \neq 0, \quad \check{\varphi}_{6}=0, \quad \check{\varphi}_{5}=0, \quad \check{\varphi}_{4}=0, \quad \check{\varphi}_{3} \neq 0, \quad \check{\varphi}_{2}=0, \quad \check{\varphi}_{1}=0, \quad \check{\varphi}_{0}=0,
$$

where the Hodge duality $\check{\varphi}_{k}=\star \varphi_{7-k}$ is utilized.

If we use the results from [1] and given $\iota \in \mathbb{Z}$,

$$
B\left(\psi, \gamma_{\alpha_{1} \ldots \alpha_{k}} \psi\right)=\left\{\begin{array}{r}
B\left({ }^{(\mathrm{Re})} \psi, \gamma_{\alpha_{1} \ldots \alpha_{k}}{ }^{(\operatorname{Im})} \psi\right)+B\left({ }^{(\operatorname{Im})} \psi, \gamma_{\alpha_{1} \ldots \alpha_{k}}{ }^{(\mathrm{Re})} \psi\right), \\
B\left({ }^{(\operatorname{Re})} \psi,\left(J \circ \gamma_{\alpha_{1} \ldots \alpha_{k}}\right)^{(\operatorname{Re})} \psi\right)-B\left({ }^{(\operatorname{Im})} \psi,\left(J \circ \gamma_{\alpha_{1} \ldots \alpha_{k}}\right)\right. \\
\text { if } k=2 \iota+1,
\end{array}\right.
$$

and

$$
B\left(\psi, J \mathbf{o} \gamma_{\alpha_{1} \ldots \alpha_{k}} \psi\right)=\left\{\begin{array}{r}
-B\left({ }^{(\operatorname{Re})} \psi, \gamma_{\alpha_{1} \ldots \alpha_{k}}{ }^{(\operatorname{Im})} \psi\right)+B\left({ }^{(\operatorname{Im})} \psi, \gamma_{\alpha_{1} \ldots \alpha_{k}}{ }^{(\mathrm{Re})} \psi\right) \\
\text { if } k=2 \iota \\
B\left({ }^{(\operatorname{Re})} \psi,\left(J \mathbf{\circ} \gamma_{\alpha_{1} \ldots \alpha_{k}}\right)^{(\mathrm{Re})} \psi\right)+B\left({ }^{(\operatorname{Im})} \psi,\left(J \mathbf{\circ} \gamma_{\alpha_{1} \ldots \alpha_{k}}\right)^{(\operatorname{Im})} \psi\right) \\
\text { if } k=2 \iota+1,
\end{array}\right.
$$

eq. (3.3) that describes the bilinear covariant coefficient of degree $k$ can be thus generalized, in order to encompass the complex case, providing the higher degree generalization of (3.2):

$$
\begin{aligned}
\beta_{k}\left(\psi, \gamma_{\alpha_{1} \ldots \alpha_{k}} \psi^{\prime}\right)=B\left({ }^{(\mathrm{Re})} \psi\right. & \left., \gamma_{\alpha_{1} \ldots \alpha_{k}}{ }^{(\mathrm{Re})} \psi^{\prime}\right)-B\left({ }^{(\mathrm{Im})} \psi, \gamma_{\alpha_{1} \ldots \alpha_{k}}{ }^{(\mathrm{Im})} \psi^{\prime}\right) \\
+ & i\left[B\left({ }^{(\mathrm{Re})} \psi, \gamma_{\alpha_{1} \ldots \alpha_{k}}{ }^{(\mathrm{Im})} \psi^{\prime}\right)+B\left({ }^{(\mathrm{Im})} \psi, \gamma_{\alpha_{1} \ldots \alpha_{k}}{ }^{(\mathrm{Re})} \psi^{\prime}\right)\right] .
\end{aligned}
$$

By using the above results, the bilinear covariants can be now extended from the standard Majorana spinor fields in $\psi \in \Gamma\left(S^{+}\right)$to sections of $\Gamma(S)$, by identifying now

$$
\varphi_{k}:=\frac{1}{k !} \beta_{k}\left(\psi, \gamma_{\alpha_{1} \ldots \alpha_{k}} \psi\right) e^{\alpha_{1} \ldots \alpha_{k}} .
$$

As both terms in the real part and also both terms in the imaginary part in (4.21) as well can cancel each other, in the complex version it is possible that the bilinears $\varphi_{0}$ and $\varphi_{4}$ can now be different from zero. Hence, four classes of spinor fields $\psi \in \Gamma(S)$ are found, concerning the classification in Riemannian 7-manifolds using the constraints above, as it is showed below:

$$
\begin{array}{ll}
\varphi_{0}=0, & \varphi_{4}=0, \\
\varphi_{0}=0, & \varphi_{4} \neq 0, \\
\varphi_{0} \neq 0, & \varphi_{4}=0, \\
\varphi_{0} \neq 0, & \varphi_{4} \neq 0 .
\end{array}
$$


It is implicit in (4.23a)-(4.23d) that all other $\varphi_{k}=0$ for $k=1,2,3,5,6,7$. Moreover, the spinor field classification according to the bilinears $\check{\varphi}_{k}$, defined by substituting eq. (4.12) in (4.21), is identical to the one provided by the $\varphi_{k}$. The above class (4.23d) encompasses the sole spinor field class (4.19), and reduces to it when we restrict the field $\psi$ to be an element of the bundle $\Gamma\left(S^{+}\right)$, namely, a Majorana spinor field.

Since $B\left(\psi, \gamma_{\alpha_{1} \ldots \alpha_{k}} \psi\right)$ vanishes except when $k \in\{0,3,4,7\}$, the graded Fierz aggregate, that is defined by

$$
\mathrm{Z}=\frac{\ell}{2^{n}} \sum_{k}(-1)^{k} B\left(\psi, \gamma_{\alpha_{1} \ldots \alpha_{k}} \psi\right) e^{\alpha_{1} \ldots \alpha_{k}},
$$

where the sum is ordered in $k$, has clearly the terms $B\left(\psi, \gamma_{\alpha_{1} \ldots \alpha_{k}} \psi\right)=0$ for such values of $k$. The above expression coincides with its Minkowski spacetime version (2.3) provided by Lounesto [18].

After classifying the spinor fields in Riemannian 7-manifolds, they can be used for defining a Lagrangian on $S^{7}$ for matter fields. According to [16], terms in a Lagrangian defined in this way depend on which realization is taken for the matter spinor fields. The classification of spinor fields in 7-manifolds can be very useful in order to study the behaviour of fields in $\mathrm{AdS}_{4} \times S^{7}$ or, more generally, in $\mathrm{AdS}_{4} \times M^{7}$, where $M^{7}$ denotes a 7-manifold.

\section{Conclusion}

Spinor fields on a manifold $(M, g)$, with arbitrary dimension and arbitrary metric signature have been classified according to the bilinear covariants. It encompasses the celebrated Lounesto's spinor field classification for Minkowski spacetime, generalizing it to arbitrary dimensions and metric signatures. The geometric Fierz identities [1] limit the amount of classes of spinor fields in such a generalized classification, which is explicitly analyzed for the important case of Majorana spinor fields on Riemannian 7-manifolds. A generalized graded Fierz aggregate is also obtained in such a context simply from the completeness relation, and we analyze the particular and prominent case of $7 \mathrm{D}$. In this case, the higher the spacetime dimension, the lesser the number of classes of spinor fields.

Despite the generalizations regarding Fierz identities were known, analysis of the spinor fields themselves had been lacking up to the middle of the last decade. Since then new models had been proposed, as for instance a candidate for the field theory of some mass dimension one fermions. A complete characterization and identification of these new spinor fields as elements of the Lounesto's classes [24] have introduced in the literature new possibilities, further investigated in e. g. $[20,21,23-28,30]$. In fact, spinor fields in the same class can present completely different dynamics. For instance, Elko spinor fields pertain to the class 5 in Lounesto's classification, and satisfy a coupled system of Dirac-like equations, whereas Majorana spinor fields are also spinor fields in the class 5, but satisfy the well-known Majorana equations. Recently the first physical example of a flag-dipole particle, which is a type- 4 spinor field in the Lounesto's classification, has been found as the solution of the Dirac equation with torsion in a $f(R)$ background [25]. Type-6 spinor 
fields encompass for instance Weyl spinor fields, but the complete dynamics of all classes is nevertheless undetermined. The first important step toward a complete characterization of all possible dynamics of spinor fields in Minkowski spacetime has been accomplished by explicitly obtaining the most general type of spinor fields in each class of Lounesto's classification [26].

Thus, the study of the Lounesto's spinor fields classification has opened a huge path to discover unexpected new physical features and to propose candidates for new particles in Minkowski spacetime [20-27, 30,32]. With this motivation we have proposed a much more general classification as well, encompassing pseudo-Riemannian spacetimes of arbitrary dimensions and metric signatures. In particular, as the subject of $S^{7}$ spinor fields is very rich [34, 37], we investigated where are the Majorana spinor fields in such a classification according to the bilinear covariants, and we concluded that the geometric Fierz identities obstruct the existence of more than one precise class, determined by (4.19), asserting that for instance that spinor fields studied in $[34,35,37]$ reside in such class. In these papers, the 3 -form bilinear is the torsion tensor that works as a gauge potential. In the most general case that we analyzed, by taking spinor fields in the spin bundle over $M$, more three types of spinor fields with potential new properties are achieved. As the singular spinor fields in Lounesto's classification were studied in exotic structures [20, 23], it is natural to relate the new classes of spinor fields in 7 -manifolds derived in (4.23a)-(4.23d) to their exotic version [53]; however this is beyond of the scope of the present paper.

\section{Acknowledgments}

R. da Rocha is grateful for the CNPq grant No. 303027/2012-6 and No. 473326/2013-2 which has provided partial support, to CAPES for the Bolsa Processo $N^{o} 10942 / 13-0$, and to SISSA for the hospitality. K. P. S. de Brito acknowledges the UFABC and CAPES grants.

Open Access. This article is distributed under the terms of the Creative Commons Attribution License (CC-BY 4.0), which permits any use, distribution and reproduction in any medium, provided the original author(s) and source are credited.

\section{References}

[1] C.I. Lazaroiu, E.M. Babalic and I.A. Coman, The geometric algebra of Fierz identities in arbitrary dimensions and signatures, JHEP 09 (2013) 156 [arXiv:1304.4403] [INSPIRE].

[2] E.-M. Babalic and C.-I. Lazaroiu, Revisiting eight-manifold flux compactifications of M-theory using geometric algebra techniques, Rom. Journ. Phys. 58 (2013) 414 [arXiv:1301.5106] [INSPIRE].

[3] S. Okubo, Representation of Clifford algebras and its applications, Math. Jap. 41 (1995) 59 [hep-th/9408165] [INSPIRE].

[4] L.S. Randriamihamison, Identites de Fierz et formes bilineaires dans les espaces spinoriels, J. Geom. Phys. 10 (1992) 19. 
[5] Y. Liao and J.-Y. Liu, Generalized Fierz identities and applications to spin-3/2 particles, Eur. Phys. J. Plus 127 (2012) 121 [arXiv:1206.5141] [INSPIRE].

[6] E.O. Korman and G. Sparling, Bilinear forms and Fierz identities for real spin representations, Adv. Appl. Clifford Algebras 22 (2012) 329 [arXiv:0901.0580] [INSPIRE].

[7] F. Reifler and R. Morris, A gauge symmetric approach to Fierz identities, J. Math. Phys. 27 (1986) 2803 [INSPIRE].

[8] Y. Takahashi, Reconstruction of spinor from Fierz identities, Phys. Rev. D 26 (1982) 2169 [INSPIRE].

[9] R.A. Mosna and J. Vaz, Quantum tomography for Dirac spinors, Phys. Lett. A 315 (2003) 418 [quant-ph/0303072] [INSPIRE].

[10] C.C. Nishi, Simple derivation of general Fierz-like identities, Am. J. Phys. 73 (2005) 1160 [hep-ph/0412245] [INSPIRE].

[11] J.F. Nieves and P.B. Pal, Generalized Fierz identities, Am. J. Phys. 72 (2004) 1100 [hep-ph/0306087] [INSPIRE].

[12] S. Rodriguez-Romo, An analysis of Fierz identities, factorization and inversion theorems, Found. Phys. 23 (1993) 1535 [INSPIRE].

[13] J. Pezzaglia, William M., Generalized Fierz identities and the superselection rule for geometric multispinors, in Spinors, twistors, Clifford algebras and quantum deformations, Z. Oziewicz et al. eds., Kluwer, (1993), gr-qc/9211018 [INSPIRE].

[14] S. Naito, K. Osada and T. Fukui, Fierz identities and invariance of eleven-dimensional supergravity action, Phys. Rev. D 34 (1986) 536 [INSPIRE].

[15] M. Baake, M. Reinicke and V. Rittenberg, Fierz identities for real Clifford algebras and the number of supercharges, J. Math. Phys. 26 (1985) 1070 [INSPIRE].

[16] H.L. Carrion, M. Rojas and F. Toppan, Quaternionic and octonionic spinors: a classification, JHEP 04 (2003) 040 [hep-th/0302113] [INSPIRE].

[17] L.V. Avdeev, On Fierz identities in noninteger dimensions, Theor. Math. Phys. 58 (1984) 203 [INSPIRE].

[18] P. Lounesto, Clifford algebras and spinors, $2^{\text {nd }}$ edition, Cambridge Univeristy Press, Cambridge U.K. (2002).

[19] A. Basak, J.R. Bhatt, S. Shankaranarayanan and K.V. Prasantha Varma, Attractor behaviour in ELKO cosmology, JCAP 04 (2013) 025 [arXiv: 1212.3445] [INSPIRE].

[20] A.E. Bernardini and R. da Rocha, Dynamical dispersion relation for ELKO dark spinor fields, Phys. Lett. B 717 (2012) 238 [arXiv:1203.1049] [InSPIRE].

[21] D.V. Ahluwalia, C.-Y. Lee, D. Schritt and T.F. Watson, Elko as self-interacting fermionic dark matter with axis of locality, Phys. Lett. B 687 (2010) 248 [arXiv:0804.1854] [INSPIRE].

[22] D.V. Ahluwalia, C.-Y. Lee and D. Schritt, Self-interacting Elko dark matter with an axis of locality, Phys. Rev. D 83 (2011) 065017 [arXiv:0911.2947] [INSPIRE].

[23] R. da Rocha, A.E. Bernardini and J.M. Hoff da Silva, Exotic dark spinor fields, JHEP 04 (2011) 110 [arXiv:1103.4759] [INSPIRE].

[24] R. da Rocha and J. Rodrigues, Waldyr Alves, Where are ELKO spinor fields in Lounesto spinor field classification?, Mod. Phys. Lett. A 21 (2006) 65 [math-ph/0506075] [INSPIRE]. 
[25] R. da Rocha, L. Fabbri, J.M. Hoff da Silva, R.T. Cavalcanti and J.A. Silva-Neto, Flag-dipole spinor fields in ESK gravities, J. Math. Phys. 54 (2013) 102505 [arXiv:1302.2262] [INSPIRE].

[26] R.T. Cavalcanti, Classification of singular spinor fields and other mass dimension one fermions, Int. J. Mod. Phys. D 23 (2014) 1444002 [arXiv:1408.0720] [inSPIRE].

[27] J.M. Hoff da Silva and R. da Rocha, Unfolding physics from the algebraic classification of spinor fields, Phys. Lett. B 718 (2013) 1519 [arXiv:1212.2406] [INSPIRE].

[28] R. da Rocha and J.M. Hoff da Silva, Hawking radiation from Elko particles tunnelling across black strings horizon, Europhys. Lett. 107 (2014) 50001 [arXiv:1408.2402] [INSPIRE].

[29] R. da Rocha and J.M. Hoff da Silva, ELKO spinor fields: lagrangians for gravity derived from supergravity, Int. J. Geom. Meth. Mod. Phys. 6 (2009) 461 [arXiv:0901.0883] [inSPIRE].

[30] R. da Rocha and J.G. Pereira, The quadratic spinor lagrangian, axial torsion current and generalizations, Int. J. Mod. Phys. D 16 (2007) 1653 [gr-qc/0703076] [INSPIRE].

[31] R. da Rocha and J.M. Hoff da Silva, ELKO, flagpole and flag-dipole spinor fields and the instanton Hopf fibration, Adv. Appl. Clifford Algebras 20 (2010) 847 [arXiv:0811.2717] [INSPIRE].

[32] M. Dias, F. de Campos and J.M. Hoff da Silva, Exploring Elko typical signature, Phys. Lett. B 706 (2012) 352 [arXiv: 1012.4642] [INSPIRE].

[33] D.V. Ahluwalia, On a local mass dimension one Fermi field of spin one-half and the theoretical crevice that allows it, arXiv:1305.7509 [INSPIRE].

[34] E. Cremmer, B. Julia and J. Scherk, Supergravity theory in eleven-dimensions, Phys. Lett. B 76 (1978) 409 [INSPIRE].

[35] F. Englert, M. Rooman and P. Spindel, Supersymmetry breaking by torsion and the Ricci flat squashed seven spheres, Phys. Lett. B 127 (1983) 47 [InSPIRE].

[36] B. Biran, F. Englert, B. de Wit and H. Nicolai, Gauged $N=8$ supergravity and its breaking from spontaneous compactification, Phys. Lett. B 124 (1983) 45 [Erratum ibid. B 128 (1983) 461] [INSPIRE].

[37] M. Cederwall and C.R. Preitschopf, $S^{7}$ and $S^{7}$ (Kac-Moody algebra), Commun. Math. Phys. 167 (1995) 373 [hep-th/9309030] [INSPIRE].

[38] R. da Rocha and J. Vaz, Jayme, Clifford algebra-parametrized octonions and generalizations, J. Algebra 301 (2011) 459 [math-ph/0603053] [INSPIRE].

[39] J.P. Crawford, On the algebra of Dirac bispinor densities: factorization and inversion theorems, J. Math. Phys. 26 (1985) 1429.

[40] R.A. Mosna and W.A. Rodrigues Jr., The bundles of algebraic and Dirac-hestenes spinor fields, J. Math. Phys. 45 (2004) 2945 [math-ph/0212033] [INSPIRE].

[41] P. Budinich, From the geometry of pure spinors with their division algebras to fermion's physics, Found. Phys. 32 (2002) 1347 [hep-th/0107158] [INSPIRE].

[42] N. Berkovits, Multiloop amplitudes and vanishing theorems using the pure spinor formalism for the superstring, JHEP 09 (2004) 047 [hep-th/0406055] [INSPIRE].

[43] N. Berkovits and P.S. Howe, Ten-dimensional supergravity constraints from the pure spinor formalism for the superstring, Nucl. Phys. B 635 (2002) 75 [hep-th/0112160] [INSPIRE]. 
[44] W.A. Rodrigues and E. Capelas de Oliveira, The many faces of Maxwell, Dirac and Einstein equations: a Clifford bundle approach, Lect. Notes Phys. 722 (2007) 1.

[45] L. Bonora, F.F. Ruffino and R. Savelli, Revisiting pinors, spinors and orientability, arXiv:0907.4334 [INSPIRE].

[46] C.-I. Lazaroiu and E.-M. Babalic, Geometric algebra techniques in flux compactifications (II), JHEP 06 (2013) 054 [arXiv: 1212.6918] [INSPIRE].

[47] I. Benn and R. Tucker, An introduction to spinors and geometry with applications in physics, Adam Hilger, Bristol U.K. (1987).

[48] S. Okubo, Real representations of finite Clifford algebras. 1. Classification, J. Math. Phys. 32 (1991) 1657 [INSPIRE].

[49] D.V. Alekseevsky, V. Cortés, Classification of N-(super)-extended Poincaré algebras and bilinear invariants of the spinor representation of $\operatorname{Spin}(p, q)$, math/9511215.

[50] P. Kaste, R. Minasian and A. Tomasiello, Supersymmetric M-theory compactifications with fluxes on seven-manifolds and G structures, JHEP 07 (2003) 004 [hep-th/0303127] [INSPIRE].

[51] K. Behrndt and C. Jeschek, Fluxes in M-theory on 7-manifolds: $G_{2}$, SU(3) and $\mathrm{SU}(2)$-structures, hep-th/0406138 [INSPIRE].

[52] T. House and A. Micu, M-theory compactifications on manifolds with $G_{2}$ structure, Class. Quant. Grav. 22 (2005) 1709 [hep-th/0412006] [INSPIRE].

[53] K. Yamagishi, Supergravity on seven-dimensional homotopy spheres, Phys. Lett. B 134 (1984) 47 [INSPIRE]. 